\title{
Detecting a Regularity in the Generation and Utilization of Primes in the Multiplicative Number Theory
}

\author{
Silviu Guiasu \\ Department of Mathematics and Statistics, York University, Toronto, Canada
}

Correspondence to: Silviu Guiasu, guiasus@pascal.math.yorku.ca

Keywords: Goldbach's Conjecture, Symmetric Prime Cousins, Systemic Approach in Number Theory, Parallel System Covering Integers with Primes, Euler's Formula for the Product of Reciprocals of Primes, Formula for the Exact Number of Primes Less than or Equal to an Arbitrary Bound

Received: May 15, 2019 Accepted: June 22, $2019 \quad$ Published: June 25, 2019

Copyright (c) 2019 by author(s) and Scientific Research Publishing Inc.

This work is licensed under the Creative Commons Attribution International License (CC BY 4.0).

http://creativecommons.org/licenses/by/4.0/

\section{(c) (i) Open Access}

\section{ABSTRACT}

If Goldbach's conjecture is true, then for each prime number $p$ there is at least one pair of primes symmetric with respect to $p$ and whose sum is $2 p$. In the multiplicative number theory, covering the positive integers with primes, during the prime factorization, may be viewed as being the outcome of a parallel system which functions properly if and only if Euler's formula of the product of the reciprocals of the primes is true. An exact formula for the number of primes less than or equal to an arbitrary bound is given. This formula may be implemented using Wolfram's computer package Mathematica.

\section{INTRODUCTION}

The prime numbers are the positive integers that can be divided only by 1 and by themselves. They are the building elements in the multiplicative number theory because every positive integer larger than 1 is a unique product of primes. The primes may be interpreted as being the "letters" of an alphabet and every positive integer larger than 1 is a "word" written with such prime letters. With such an interpretation, all positive integers are "written" with, or "covered" by sequences of primes. For instance, we have $100=2^{2} \times 5^{2}=[2,2 ; 2,5]$ where the last notation indicates the powers of the corresponding prime factors of the integer 100. Similarly, $78936=2^{3} \times 3 \times 11 \times 13 \times 23=[3,1,1,1,1 ; 2,3,11,13,23]$, and $63992=[3,1,1 ; 2,19,421]$ or $1000=[4,4 ; 2,5]$. In this prime codification of the positive integers, the primes are the only one-letter words, such as $5=[1 ; 5]$, for instance.

In the sequence of positive integers, the primes appear randomly and the gap between consecutive primes fluctuates without an obvious pattern. There is, however, the spread belief that there is some hidden regularity in the generation of primes and in their utilization in the multiplicative covering of the positive integers with primes. In this paper, it is proved that there is a certain symmetry in the location of primes in the sequence of positive integers. Also, the process of covering positive integers with primes, as 
stated by the unique prime factorization, may be viewed as the outcome of a parallel system which functions properly if and only if Euler's formula for the product of reciprocals of prime numbers holds. Finally, an exact formula for the number of primes less than or equal to an arbitrary bound is given. This formula may be implemented using Wolfram's computer package Mathematica.

\section{SYMMETRY}

If $x_{1}$ and $x_{2}$ are real numbers whose sum is $S=x_{1}+x_{2}$ and product is $P=x_{1} x_{2}$, then they are solutions of the second degree equation $x^{2}-S x+P=0$. If $n$ is a positive integer number such that $2 n=p+q$, where $p$ and $q$ are primes, then $p$ and $q$ are symmetric with respect to $n$, which means that $n$ is at the middle distance between $p$ and $q$. Indeed, $p$ and $q$ are solutions of the above second degree equation with $S=p+q=2 n$, and $P=p q$, namely: $x^{2}-2 n+p q=0$. Thus,

$$
\begin{aligned}
& p=n-\sqrt{n^{2}-p q}=n-(1 / 2)(q-p), \\
& q=n+\sqrt{n^{2}-p q}=n+(1 / 2)(q-p)
\end{aligned}
$$

which show that the primes $p$ and $q$ are symmetric with respect to $n$.

In the margins of a letter sent to Leonhard Euler on 7 June 1742, Christian Goldbach wrote that every even integer greater than 4 is the sum of two primes. More than one such pair of primes could exist. For instance: $100=3+97=11+89=17+83=29+71=41+59=47+53$. But there is no proof that such a pair of primes does exist for every even integer greater than 4. If Goldbach's conjecture is true, then to each prime number $n$ there is at least one pair of primes $p$ and $q$ such that $2 n=p+q$ and, according to (1), they are symmetric with respect to $n$, locate at a distance equal to $\pm(1 / 2)(q-p)$ from the prime number $n$. Two primes $p$ and $q$ symmetric with respect to the prime $n$, such that $p+q=2 n$ are called prime cousins of the prime number $n$. Thus, if Goldbach's conjecture is true, then every prime number larger than 3 has at least one pair of prime cousins. For instance, 3 and 7 are prime cousins of 5;29 and 53 are prime cousins of 41;59 and 83 are prime cousins of 71, and so on.

\section{SYSTEMIC APPROACH}

\subsection{Brief System Theory}

Let $S$ be a system which consists of $k$ binary components. The $i$-th component functions properly with probability $q_{i}$ and fails to function properly with probability $1-q_{i}$, where $q_{i}$ is a real number from the interval $[0,1]$. The structure function of the $i$-th component is a binary random variable $f_{i}$ equal to 1 (success) if the $i$-th component functions properly, which happens with probability $q_{i}$, and equal to 0 (failure) if the $i$-th component fails to function properly, which happens with probability $1-q_{i}$. Generally, the $i$-th component is identified with its structure function $f_{i}$. Reliability of the $i$-th component is the probability that this component functions properly or, equivalently, probability that the structure function $f_{i}$ is equal to 1 . Therefore, $R_{i}=q_{i}=P\left(f_{i}=1\right)$.

The system $S$ is a series system if it functions properly if and only if each of its components functions properly. It fails to function properly if at least one of its components fails to function properly. The structure function of a series system is ([1], pp.190-203):

$$
f=\min \left\{f_{1}, \cdots, f_{k}\right\}=f_{1} \cdots f_{k} .
$$

The series system $S$ is identified with its structure function $f$ given by (1). As the components are independent, the reliability of the series system is the probability that the system functions properly, which is:

$$
R=P(f=1)=P\left(f_{1}=1, \cdots, f_{k}=1\right)=P\left(f_{1}=1\right) \cdots P\left(f_{k}=1\right)=R_{1} \cdots R_{k} .
$$

The system $S$ is a parallel system if it fails to function properly if and only if each of its components fails to function properly. It functions properly if at least one of its components functions properly. The structure function of a parallel system is: 


$$
f=\max \left\{f_{1}, \cdots, f_{k}\right\}=1-\left(1-f_{1}\right) \cdots\left(1-f_{k}\right) .
$$

The parallel system $S$ is identified with its structure function $f$ given by (2). As the components are independent, the reliability of the parallel system is the probability that the system functions properly, which is:

$$
\begin{aligned}
& 1-P\left(f_{1}=0, \cdots, f_{k}=0\right) \\
& =1-P\left(f_{1}=0\right) \cdots P\left(f_{k}=0\right) \\
& =1-\left[1-P\left(f_{1}=1\right)\right] \cdots\left[1-P\left(f_{k}=1\right)\right] \\
& =1-\left(1-R_{1}\right) \cdots\left(1-R_{k}\right)
\end{aligned}
$$

\subsection{The Parallel System Performing the Prime Factorization of Integers}

The general system theory allows us to see that periodic binary independent components of a parallel system cover multiplicatively all the positive integers larger than 1 with primes or integer powers of primes. Remarkably, the reliability of this parallel system, measuring the efficiency of the covering process, is given by Euler's formula of the product of the reciprocals of the prime numbers.

A component system analyzes how well the natural numbers are covered by the prime number $p$ in the multiplicative number theory. It examines sequentially, one by one, the set of positive integers. When the examined integer is the prime number $p$ or one of its multiples, the search is a success; if not, the search is a failure. The structure function $X_{p}$ of such a component system takes on the value 1 (success) when the system finds the prime number $p$ or one of its multiples, and the value 0 (failure) when the examined integer is not the prime number $p$ or one of its multiples. The structure function $X_{p}$ is periodic, with the period of length $p$. In the first period, for instance, the structure function $X_{p}$ assigns the value 0 (failure) to the integer numbers $1,2, \cdots, p-1$, and the value 1 (success) to the last integer $p$ from the first period. In the second period, the structure function $X_{p}$ assigns the value 0 (failure) to the integer numbers $p+1, p+2, \cdots, 2 p-1$, and the value 1 (success) to the last integer $2 p$ from the second period. And so on. Taking into account what happens in these periods, the structure function $X_{p}$ may be identified with a binary random variable whose value 1 (success) has the probability $1 / p$, and the possible value 0 (failure) has the probability $1-1 / p$.

$$
X_{p}=\left(\begin{array}{cc}
1 & 0 \\
\frac{1}{p} & 1-\frac{1}{p}
\end{array}\right)
$$

Reliability of the component system with the structure function $X_{p}$ is the probability that the component system is successful, namely:

$$
R\left(X_{p}\right)=P\left(X_{p}=1\right)=\frac{1}{p} .
$$

The number $R\left(X_{p}\right)$ measures the efficiency of "covering" positive integers with the multiples of the prime number $p$. Probability that the component system fails to find a multiple of $p$ is:

$$
1-R\left(X_{p}\right)=P\left(X_{p}=0\right)=1-\frac{1}{p} .
$$

Let $S_{1}$ be the system obtained by joining in parallel the component systems $\left\{X_{p}, p \in \mathbb{P}\right\}$, where $\mathbb{P}$ is the set of prime numbers. The reliability of the parallel system $S_{1}$ is:

$$
R\left(S_{1}\right)=P\left(S_{1}=1\right)=1-\prod_{p}\left[1-R\left(X_{p}\right)\right]=1-\prod_{p}\left(1-\frac{1}{p}\right)=1,
$$


showing that the efficiency of "covering" the positive integers larger than 1 with prime numbers is maximum, because, according to Euler's product formula $([2,3])$, we have:

$$
1-\prod_{p}\left(1-\frac{1}{p}\right)=1-\frac{1}{\zeta(1)},
$$

where Euler's zeta function has the value $\zeta(1)=\infty$, being the sum of the harmonic series. It is remarkable that Euler's product formula appears in the expression of the reliability of the parallel system $S_{1}$. In fact, the equality (3) may be obtained independently of Euler's product formula. Indeed, the parallel system $S_{1}$ properly assigns a prime number to every positive integer larger than 1 and, according to the fundamental theorem of arithmetic, every positive integer larger than 1 is either a prime number or the product of prime numbers. Therefore, each positive integer larger than 1 has at least one prime number assigned to it. Consequently, the probability that the parallel system $S_{1}$ fails to assign prime numbers to the positive integers larger than 1 is equal to zero, namely:

$$
P\left(S_{1}=0\right)=\prod_{p}\left(1-\frac{1}{p}\right)=0,
$$

which is equivalent to (3).

If $s$ is a positive integer number, let $X_{p^{s}}$ be the structure function of the component system which analyzes how well the natural numbers are multiplicatively covered by the $s$-th power of the prime number $p$. It examines sequentially, one by one, the set of positive integers. When the examined integer is the number $p^{s}$ or one of its multiples, the search is a success; if not, the search is a failure. The structure function $X_{p^{s}}$ of such a component system takes on the value 1 (success) when the system finds the number $p^{s^{s}}$ or one of its multiples, and the value 0 (failure) when the examined integer is not the number $p^{s}$ or one of its multiples. The structure function $X_{p^{s}}$ is periodic, with periods of length $p^{s}$. In the first period, for instance, the structure function $X_{p^{s}}$ assigns the value 0 (failure) to the positive integers $1,2, \cdots, p^{s}-1$, and the value 1 (success) to the last integer $p^{s}$ from the first period. In the second period, the structure function $X_{p^{s}}$ assigns the value 0 (failure) to the integer numbers $p^{s}+1, p^{s}+2, \cdots, 2 p^{s}-1$, and the value 1 (success) to the last integer $2 p^{s}$ from the second period. And so on. Taking into account what happens in these periods, the structure function $X_{p^{s}}$ may be identified with a binary random variable whose possible value 1 (success) has the probability $p^{s} 1 / p^{s}$, and the possible value 0 (failure) has the probability $1-1 / p^{s}$. Thus:

$$
X_{p^{s}}=\left(\begin{array}{cc}
1 & 0 \\
\frac{1}{p^{s}} & 1-\frac{1}{p^{s}}
\end{array}\right) .
$$

Reliability of the component system with the structure function $X_{p^{s}}$ is the probability that the component system finds a success, namely:

$$
R\left(X_{p^{s}}\right)=P\left(X_{p^{s}}=1\right)=\frac{1}{p^{s}} .
$$

Probability that the component system fails to find a success is:

$$
1-R\left(X_{p^{s}}\right)=P\left(X_{p^{s}}=0\right)=1-\frac{1}{p^{s}} .
$$

Let $S_{s}$ be the system obtained by joining in parallel the component systems $\left\{X_{p^{s}}, p \in \mathbb{P}\right\}$, where $\mathbb{P}$ is the set of prime numbers. Using Euler's product formula $([2,3])$, the reliability of the parallel system $S_{s}$ is: 


$$
R\left(S_{s}\right)=P\left(S_{s}=1\right)=1-\prod_{p}\left[1-R\left(X_{p^{s}}\right)\right]=1-\prod_{p}\left(1-\frac{1}{p^{s}}\right)=1-\frac{1}{\zeta(s)},
$$

where $\zeta(s)$ is Euler's zeta function.

Probability that the parallel system $S_{s}$ fails to find the $s$-th power of a prime or a multiple of the $s$-th power of a prime is:

$$
1-R\left(S_{s}\right)=P\left(S_{s}=0\right)=\prod_{p}\left(1-\frac{1}{p^{s}}\right)=\frac{1}{\zeta(s)} .
$$

In particular, it follows from (4) that the efficiency of "covering" the positive integers larger than 1 with powers of primes of order 2, 3, or 4 is given by the numbers:

$$
\begin{aligned}
& R\left(S_{2}\right)=1-\frac{1}{\zeta(2)}=1-\frac{6}{\pi^{2}} \cong 0.3921, \\
& R\left(S_{3}\right)=1-\frac{1}{\zeta(3)} \cong 0.1681, \\
& R\left(S_{4}\right)=1-\frac{1}{\zeta(4)}=1-\frac{90}{\pi} \cong 0.0760 .
\end{aligned}
$$

\section{COUNTING THE DENSITY OF PRIMES}

\subsection{Previous Attempts}

In the set of positive integer numbers, the prime numbers (i.e. the numbers that can be exactly divided only by 1 and by themselves) appear in a rather irregular way. Denoting by $\pi(x)$ the number of primes not exceeding the positive real number $x$, there are well-known formulas that approximate it, as mentioned in [4], for instance. Some of these approximations are quite ingenious but are there exact formulas for $\pi(x)$ ? The answer is yes and the simplest formula was given by Ján Minác. Apparently, he did not publish it but his formula is given on page 133 of the book [5], with a simple proof based on a very old result obtained by John Edward Wiilson in 1770, and attributed even to Ibn al-Haytham (c. 1000 AD), stating that for any prime $j,(j-1) !+1=k j$, where $k$ is an integer and the factorial $(j-1) !=1 \times 2 \times 3 \times \cdots \times(j-2) \times(j-1)$. Minác's formula is:

$$
\pi(n)=\sum_{j=1}^{n}\left[\frac{(j-1) !+1}{j}-\left[\frac{(j-1) !}{j}\right]\right] .
$$

where $n$ is an arbitrary positive integer and $[x]$ is the largest integer not exceeding the real number $x$. This formula is elegant, but difficult to use because the factorial is the most rapidly increasing function. Thus, if we want to calculate $\pi(30)$, for instance, it involves factorials up lo $29 ! \cong 8.841761993 E+30$, whereas its exact value is: $29 !=8841761993739701954543616000000$.

For larger and larger integers, even the powerful computers can only approximate their factorials until the capacity to store numbers is exhausted. Thus, Minác's formula gives the exact value of $\pi(n)$ but its applicability is very limited.

Another well-known formula involving $\pi(x)$ was given by Legendre ([6]), later refined by E.D.F. Meissel as mentioned in D.H. Lehmer [7].

It is well known that if the values of $\pi(x)$ are given, we can calculate the number $Q(x)$ of primes and integer powers of primes less than or equal to the number $x$ using the Möbius' formula:

$$
Q(x)=\pi(x)+\pi\left(x^{1 / 2}\right)+\pi\left(x^{1 / 3}\right)+\cdots+\pi\left(x^{1 / n(x)}\right),
$$


where $n(x)$ is the largest positive integer such that: $x^{1 / n(x)} \geq 2$, which is equivalent to:: $n(x) \leq\left[\log _{2} x\right]$.

In what follows, we change the approach, getting an exact formula for $Q(x)$, first, and using the inverse of the Möbius' formula (1) in order to get an exact formula for $\pi(x)$ later. As the exact formulas for $Q(x)$ and $\pi(x)$ are algorithmic formulas, the best strategy is to see how they work on a numerical example and to generalize them afterwards. The exact formulas for $Q(x)$ and $\pi(x)$ thus obtained may be implemented using Wolfram's computer package Mathematica ([8]).

\subsection{An Exact Formula for $Q(x)$}

Case \#1: Using the primes less than or equal to $x$. In order to avoid writing too much, assume that $x=30$. Using the primes less than 30 , namely $p_{1}=2,3,5,7,11,13,17,19,23,29$, we want to give a general approach of how to calculate $Q(30)$, based on common sense. First, for each prime number $p_{1}$ less than 30 , we calculate the number of its multiples not exceeding 30 , which is $\left[\frac{30}{p_{1}}\right]$, and take their sum:

$$
\begin{aligned}
S_{p_{1}}= & \sum_{p_{1}<30}\left[\frac{30}{p_{1}}\right] \\
= & {\left[\frac{30}{2}\right]+\left[\frac{30}{3}\right]+\left[\frac{30}{5}\right]+\left[\frac{30}{7}\right]+\left[\frac{30}{11}\right] } \\
& +\left[\frac{30}{13}\right]+\left[\frac{30}{17}\right]+\left[\frac{30}{19}\right]+\left[\frac{30}{23}\right]+\left[\frac{30}{29}\right] \\
= & 43
\end{aligned}
$$

For each pair of prime numbers $p_{1}<p_{2}<30$, we calculate the number of the multiples of $p_{1} \times p_{2}$ not exceeding 30 , which is $\left[\frac{30}{p_{1} \times p_{2}}\right]$, and take their sum:

$$
\begin{aligned}
S_{p_{1} \times p_{2}}= & \sum_{p_{1} \times p_{2}<30 ; p_{1}<p_{2}}\left[\frac{30}{p_{1} \times p_{2}}\right] \\
= & {\left[\frac{30}{2 \times 3}\right]+\left[\frac{30}{2 \times 5}\right]+\left[\frac{30}{2 \times 7}\right]+\left[\frac{30}{2 \times 11}\right] } \\
& +\left[\frac{30}{2 \times 13}\right]+\left[\frac{30}{3 \times 5}\right]+\left[\frac{30}{3 \times 7}\right] \\
= & 15
\end{aligned}
$$

For each triplet of prime numbers $p_{1}<p_{2}<p_{3}<30$, we calculate the number of the multiples of $p_{1} \times p_{2} \times p_{3}$, not exceeding 30 , which is $\left[\frac{30}{p_{1} \times p_{2} \times p_{3}}\right]$, and take their sum:

$$
S_{p_{1} \times p_{2} \times p_{3}}=\sum_{p_{1} \times p_{2} \times p_{3}<30 ; p_{1}<p_{2}<p_{3}}\left[\frac{30}{p_{1} \times p_{2} \times p_{3}}\right]=\left[\frac{30}{2 \times 3 \times 5}\right]=1 .
$$

We stop here because no product of four primes less than 30 is itself less than or equal to 30 . Both the multiples of $p_{1}$ and the multiples of $p_{2}$ contain the multiples of $p_{1} \times p_{2}$. Also, both the multiples of $p_{1}$ and the multiples of $p_{2}$ and the multiples of $p_{3}$ contain the multiples of $p_{1} \times p_{2} \times p_{3}$. Finally, the multiples of $p_{1} \times p_{2}$ contain the multiples of $p_{1} \times p_{2} \times p_{3}$. Therefore, the number of primes and integer powers of primes less than or equal to 30 is: 


$$
Q(30)=S_{p_{1}}-2 S_{p_{1} \times p_{2}}+3 S_{p_{1} \times p_{2} \times p_{3}}=16 .
$$

Taking an arbitrary real number $x$ and following the same steps as mentioned in the above example, we have the general formula:

$$
Q(x)=\sum_{r \geq 1}(-1)^{r+1} r \sum_{p_{1}<\cdots<p_{r} ; p_{1} \times \cdots \times p_{r} \leq x}\left[\frac{x}{p_{1} \times \cdots \times p_{r}}\right] .
$$

Case \#2: Using only the prime factorizations of the integers less than or equal to $x$ that contain no powers of primes. This case is very similar to Case \#1 but it assumes more information about the integers not exceeding $x$. It allows, however, to get an equivalent formula for $Q(x)$ which may be implemented on the computer.

We want to evaluate again the value of $Q(30)$ but assuming this time that we know the prime factorizations of the positive integers up to 30 that contain no powers of primes, namely:

$$
\begin{gathered}
2=2,3=3,5=5,6=2 \times 3,7=7,10=2 \times 5,11=11, \\
13=13,14=2 \times 7,15=3 \times 5,17=17,19=19,21=3 \times 7, \\
22=2 \times 11,23=23,26=2 \times 13,29=29,30=2 \times 3 \times 5 .
\end{gathered}
$$

We do now what we did in Case \#1. For the numbers whose prime factorizations contain only one prime, namely for: $n=2,3,5,7,11,13,17,19,23,29$, the numbers of their multiples not exceeding 30 are:

$$
\left[\frac{30}{n}\right]=15,10,6,4,2,2,1,1,1,1
$$

respectively, and their sum is just: $S_{p_{1}}=43$.

For the numbers whose prime factorizations contain only two distinct primes, namely for:

$$
n=6,10,14,15,21,22,26
$$

the numbers of their multiples not exceeding 30 are:

$$
\left[\frac{30}{n}\right]=5,3,2,2,1,1,1
$$

respectively, and their sum is just: $S_{p_{1} \times p_{2}}=15$.

For the numbers whose prime factorizations contain only three distinct primes, namely for $n=30$, the number of its multiples not exceeding 30 is just:

$$
S_{p_{1} \times p_{2} \times p_{3}}=\left[\frac{30}{n}\right]=1 .
$$

As already mentioned in Case \#1, both the multiples of $p_{1}$ and the multiples of $p_{2}$ contain the multiples of $p_{1} \times p_{2}$. Also, both the multiples of $p_{1}$ and the multiples of $p_{2}$ and the multiples of $p_{3}$ contain the multiples of $p_{1} \times p_{2} \times p_{3}$ Finally, the multiples of $p_{1} \times p_{2}$ contain the multiples of $p_{1} \times p_{2} \times p_{3}$. Therefore, the number of primes and powers of primes less than 30 is:

$$
Q(30)=S_{p_{1}}-2 S_{p_{1 \times p_{2}}}+3 S_{p_{1} \times p_{2} \times p_{3}}=16 .
$$

In order to apply this approach to calculate $Q(x)$ for an arbitrary positive number $x$, we have to get rid of those integers $n \leq x$ whose prime factorizations contain powers of primes and to assign alternating signs to the sums of multiples of the remaining integers $n \leq x$, as we did in (7). Fortunately, there is a magic function which does these things, introduced long ago, in 1831 in fact, by Augustus Ferdinand Möbius. This is the same mathematician who described the Möbius strip, a famous two-dimensional surface with only one side. The Möbius function is defined on the set of positive integers by: 


$$
\mu(n)= \begin{cases}1, & \text { if } n=1 ; \\ 0, & \text { if } n \text { is divisible by a square of a prime } p \\ (-1)^{r}, & \text { if } n=p_{1} \times \cdots \times p_{r} \text { for distinct primes } p_{1}, \cdots, p_{r} .\end{cases}
$$

Using the Möbius function $\mu$ and the factor function $\Omega$ which gives the total number of prime factors of the positive integers (for instance, $\Omega(540)=\Omega\left(2^{2} \times 3^{3} \times 5\right)=6$ ) we can remake the steps followed for calculating $Q(30)$ in Case \#2 and we obtain the formula:

$$
Q(x)=-\sum_{n=2}^{[x]} \mu(n) \Omega(n)\left[\frac{x}{n}\right] .
$$

In this formula, the Möbius function $\mu$ chooses only those integers $n$ whose prime factorizations contain no powers of primes and assigns the alternative signs + or - , whereas the factor function $\Omega$ takes care of the integer coefficients representing the number of prime factors from the factorizations of the remaining integers $n$, as we did when we dealt with the numerical result (7) from Case \#2.

\subsection{An Exact Formula for $\pi(x)$}

Applying the well-known inverse of the Möbius formula (5), we get:

$$
\pi(x)=\sum_{k=1}^{\left[\log _{2} x\right]} \mu(k) Q\left(x^{1 / k}\right)
$$
ber $x$.

From (8) and (9) we get the exact formula for the number of primes not exceeding the positive num-

$$
\pi(x)=-\sum_{k=1}^{\left[\log _{2} x\right]} \mu(k) \sum_{n=2}^{\left[x^{1 / k}\right]} \mu(n) \Omega(n)\left[\frac{x^{1 / k}}{n}\right] .
$$

\subsection{Computer Assistance}

Formulas (8) and (10) may be implemented using Stephen Wolfram's computer package Mathematica [8]. Using the version Mathematica 2.1 on Windows, we type the following commands: At the end of each command we choose from the top bar "Action" and click on "Evaluate Selection".

Command 1:

$\mathrm{a}\left[\mathrm{x}_{-}\right]:=$Floor $[\mathrm{N}[\mathrm{x}]]$

Command 2:

$\mathrm{f}\left[\mathrm{x}_{-}\right]:=-$Sum $\left[\right.$MoebiusMu[n] ${ }^{\star}$ Apply[Plus,Transpose[FactorInteger[n]][[2]]]*Floor[x/n],

$\{\mathrm{n}, 2, \mathrm{a}[\mathrm{x}], 1\}]$

Command 3:

$\mathrm{b}\left[\mathrm{x}_{-}\right]:=$Floor $[\mathrm{N}[\log [2, \mathrm{x}]]]$

Command 4:

$\mathrm{h}\left[\mathrm{x}_{-}\right]:=\mathrm{N}\left[\operatorname{Sum}\left[\right.\right.$ MoebiusMu$\left.\left.[\mathrm{k}]^{\star} \mathrm{f}\left[\mathrm{x}^{\wedge}(1 / \mathrm{k})\right],\{\mathrm{k}, 1, \mathrm{~b}[\mathrm{x}], 1\}\right]\right]$

If we want to get the values of $Q(30)$ and $\pi(30)$ we type the commands:

Command 5:

$\mathrm{f}[30]$

Command 6:

$\mathrm{h}[30]$

and we get the answers 16 and 10, respectively. Similarly, if we want to get the values of $Q(10000)$ and $\pi(10000)$, we type the commands:

Command 7:

f[10000] 
Command 8:

$\mathrm{h}[10000]$

and we get the answers 1280 and 1229 , respectively.

Remark: Mathematica has two practical commands: Prime[n], which gives the $\mathrm{n}$-th prime, and PrimePi[x], which gives the value of $\pi(x)$, but we do not know how these values are calculated by this computer package. The details are kept secret.

\section{CONCLUSIONS}

The objective is to find some regularity in the generation and utilization of primes in the multiplicative number theory. In Section 2 it is shown that if Goldbach's conjecture is true, then for every prime $n$, larger than 3, there is at least one pair of primes symmetric with respect $n$.

Section 3 contains a systemic approach of the prime factorization of the positive integers larger than 1. In the multiplicative number theory, the prime factorization of the integers is viewed as the outcome of a system which analyzes how well the natural numbers are covered by the prime number $p$. It examines sequentially, one by one, the set of positive integers. When the examined integer is the prime number $p$ or one of its multiples, the search is a success; if not, the search is a failure. The structure function $X_{p}$ of such a component system takes on the value 1 (success) when the component system finds the prime number $p$ or one of its multiples, and the value 0 (failure) when the examined integer is not the prime number $p$ or one of its multiples. The structure function $X_{p}$ is periodic, with periods of length $p$. In the first period, for instance, the structure function $X_{p}$ assigns the value 0 (failure) to the integers $1,2, \cdots, p-1$, and the value 1 (success) to the last integer $p$ from the first period. In the second period, the structure function $X_{p}$ assigns the value 0 (failure) to the integer numbers $p+1, p+2, \cdots, 2 p-1$, and the value 1 (success) to the last integer $2 p$ from the second period. And so on. Taking into account what happens in these periods, the structure function $X_{p}$ may be identified with a binary random variable whose possible value 1 (success) has the probability $1 / p$, and the possible value 0 (failure) has the probability $1-1 / p$. The reliability $R\left(X_{p}\right)$, showing how efficiently the prime number $p$ covers the positive integers, is $1 / p$. The parallel connection of the component systems $\left\{X_{p}, p \in \mathbb{P}\right\}$, where $\mathbb{P}$ is the set of prime numbers, describes how the set of all prime numbers covers the set of positive integers larger than 1 . Euler's well-known formula for the product of the reciprocals of the primes implies that the reliability of this parallel system is maximum and equal to 1 , in agreement with the fundamental theorem of arithmetic. The same analysis was done to calculate how efficiently the integer powers of prime numbers cover the set of positive integers larger than 1.

In Section 4, formula (6) gives the number of primes and integer powers of primes not exceeding the arbitrary real number $x$ using only the primes numbers less than or equal to $x$. Formula (8) gives the number of primes and integer powers of primes not exceeding the arbitrary real number $x$ using the prime factorization of the positive integers not exceeding $x$. Formula (10) gives the number of primes not exceeding the arbitrary positive real number $x$. Formulas (8) and (10) may be implemented using Wolfram's computer package Mathematica [8] for Windows.

Summarizing, the original contributions of this paper are the following ones: 1) To introduce the concept of symmetric prime cousins of a prime number; 2) To use a systemic approach according to which the processor of covering the positive integers larger than 1 with primes in the multiplicative number theory may be viewed as the outcome of a parallel system. Surprisingly, the efficiency of this process is measured by Euler's formula for the product of the reciprocals of the prime numbers; 3) Exact formulas are given for the number of primes and for the number of integer powers of primes less than or equal to an arbitrary real bound. These formulas may be implemented using Wolfram's computer package Mathematica for Windows.

\section{CONFLICTS OF INTEREST}

The authors declare no conflicts of interest regarding the publication of this paper. 


\section{REFERENCES}

1. Guiasu, S. (2009) Probabilistic Models in Operations Research. Nova Science Publishers, New York.

2. Hardy, G.H. and Wright, E.M. (1962) Introduction to the Theory of Numbers. 4th Edition, Clarendon Press, Oxford.

3. Guy, R.K. (1981) Unsolved Problems in Number Theory. Springer-Verlag, New York-Heidelberg-Berlin.

4. Guiasu, S. (1995) Is There Any Regularity in the Distribution of Prime Numbers at the Beginning of the Sequence of Positive Integers? Mathematics Magazine, 68, 110-121.

https://doi.org/10.1080/0025570x.1995.11996292

5. Ribenboim, P. (2004) The Little Book of Bigger Primes. 2nd Edition, Springer-Verlag, New York.

6. Legendre, A.-M. (1830) Théorie des Nombres. 3rd Edition, Firmin Didot Frères, Paris.

7. Lehmer, D.H. (1959) On Exact Number of Primes Less Than a Given Bound. Illinois Journal of Mathematics, 3 , 381-388. https://doi.org/10.1215/ijm/1255455259

8. Wolfram, S. (1991) Mathematica. A System of Doing Mathematics by Computer. 2nd Edition, Addison Wesley Publishing Company, Redwood City, California. 